\title{
THE USE OF VIRTUALIZATION TECHNOLOGY TO SUPPORT INFORMATION TECHNOLOGY PROGRAMMING COURSES
}

\author{
George Stefanek, Purdue University Northwest, stefanek@pnw.edu
}

\begin{abstract}
The use of virtualization technology and the issues encountered using such an environment in the support of undergraduate information technology programming courses is presented in this paper. A Virtual Desktop Infrastructure (VDI) platform hosted by a university IS datacenter is a technology that can overcome many of the issues encountered when students configure their own computers with multiple software packages that are required for advanced programming courses. Some of the problems that students encounter when installing an Integrated Development Environment and database system on their own computers range from installation problems to compatibility issues when integrating software developed on different computers. Also, some students do not have their own computers. VDI environments have the software pre-installed and allow for teams of students to collaborate using identical software at the same revision level. This paper presents finding on the issues encountered when using a server-based VDI platform and compares the results to a previous study in the same courses using the same software where students installed their own software on their own computers.
\end{abstract}

Keywords: Virtualization, VDI, Virtualization Platform, Virtual Machines, Educational Technology

\section{INTRODUCTION}

Computer Information Technology departments at universities often require that students in advanced programming courses develop computer code that interfaces with a database management system. This type of code development requires that an Integrated Development Environment (IDE) and a database management system (DBMS) be installed on the computers which students use to do their projects. Most students try to install these software packages (i.e., the IDE and DBMS) on their own computers so that they have the flexibility to work on their homework and projects at any time of the day or night. The IDEs used at the university in information technology courses include Microsoft Visual Studio, Netbeans and Android Studio. The DBMS systems include MySQL, Microsoft SQL Server and ORACLE Database.

Students typically configure their own computers with the required software for a course. The software is either downloaded from the internet or from university resources. Some students have problems finding the correct versions of the required software to download from the internet and occasionally run into problems installing the software such as compatibility issues and lack of resources. Finally, some courses require team projects at the end of the semester where students work in teams to develop real-world-like applications. Stefanek and Patel (2015) show that when students install software themselves on their personal computers they often encounter numerous problems integrating different pieces of software from other student team members into a single application. Mostly, these problems are due to students having different versions of the IDE or different versions of the database management system installed on their own computers.

Some students do not have their own personal computers and therefore cannot install the required software for the course. These students must use university lab computers. Students often work late at night and need to have 24/7 access to the required programming environments even when the university computer labs are closed. Other students have their own personal computers, but do not have adequate hardware to run the required software adequately. Examples of under configured hardware are too little RAM, slow CPUs, not enough space on hard disk drives, and outdated BIOS. 
Additionally, students need to persist their databases in programming classes which is not possible on university lab computers. Lab computers load a new image of the system every time the lab computer is rebooted. Backing up and restoring the database every time they use the lab computers is time-consuming and frustrating for users.

Finally, students need to have computers with compatible software packages at the same version level when integrating code on team projects. When students configure their own computers, the software may vary from computer to computer. Students need to have access to software development environments that have the appropriate software installed and necessary hardware resources to do their programming projects. Sometimes students do not have the adequate hardware resources to install all the required software, have difficulty installing the required software, may not install the correct software, and may not install the software at the specified version level. Even though virtual machines (VMs) (VMware, 2015) can be preconfigured with the required software for specific programming courses and then distributed directly to students, students may not be able to run the VMs locally on their personal computers and may experience poor VM performance. Also, students may not know which version of virtual machine software to download such as VMware Workstation (VMWare, 2017 -c) or Oracle VM Virtualbox (Oracle, 2017), may not know how to configure the virtual machine software, and may not know how to configure their own computer hardware to support the running of virtual machines.

A Virtual Desktop Infrastructure (VDI) platform hosted on servers at the university IS datacenter is a technology that can overcome many of the issues encountered by students when configuring their own computers with multiple software packages required for advanced programming courses. Citrix (2017) defines a Virtual Desktop Infrastructure (VDI) as the process of running a user desktop inside a virtual machine that resides on a server in a datacenter. It is a form of virtualization (VMWare, 2017 -b) that enables personalized desktops for each user to be run within a virtual machine, but with all the security and simplicity of centralized management. Since multiple VMs can be run on a single server, this approach enables students to streamline management and costs by consolidating and centralizing the desktops while delivering students $24 / 7$ access to the virtual desktops wherever there is a computer with an internet connection. Additionally, the server-based VDI platform allows all students in a class to access pre-configured VDIs that have the required software installed for each specific information technology course and provides access to the environment $24 / 7$ both on-site and off-site.

This paper presents finding on the issues encountered when using a server-based Virtual Desktop Infrastructure platform in the teaching of two undergraduate programming courses and compares the results of these experiences to a previous study in the same courses where students installed the required software on their own computers. The software used in the courses was the same in this study and the previous study.

\section{VIRTUALIZATION TECHNOLOGY BACKGROUND}

A virtual machine (VM) is a software computer that like a physical computer runs an operating system and can execute its applications (VMware, 2015). Bauer, E., \& Adams, R. (2012) describe virtualization as decoupling an application from the underlying physical hardware, including CPU, networking, memory, and disk data storage. Application software is said to be virtualized (i.e., experiences virtualization) as a virtual machine (VM) and is defined by Bauer, E., \& Adams, R. (2012) as "an encapsulation of the virtual hardware, virtual disks, and the metadata associated with it." Bauer, E. \& Adams, R. (2012) state that one of the key components of virtualization is the hypervisor which supports the multiple operating systems being run concurrently on a single host computer. The hypervisor manages the applications' operating systems and their use of the system resources such as the CPU, memory, and disk storage. Virtual machines (VMs) are isolated instances of the application software and operating system they are installed in that run as a separate computer. The hypervisor supports this isolation and manage the multiple VMs running on the same host (server) computer. Virtualization technology uses software to simulate hardware and create a virtual computer system which allows organizations to run more than one virtual computer with multiple operating systems and applications on a single server. This type of technology has the benefit of providing economies of scale and greater efficiency (VMWare, $2017-\mathrm{b})$. 
There are four major types of virtualization technology as defined by Shields, G. (2008): hardware, operating system, application virtualization, and para-virtualization. The specific technology used at the Purdue University Northwest Information Services department to host the virtual machines is VMWare Horizon with View (VMWare, 2017 -a). This technology is a hardware virtualization type technology that emulates the physical computer hardware and runs an installed operating system that is compatible with the emulated hardware. A thin layer of software (i.e., the hypervisor) is a virtual machine monitor of the isolated virtual machines running on the host server which dynamically allocates computing resources to each virtual machine as needed (VMware, 2015). As each virtual machine encapsulates an entire computer, many applications and operating systems can run on a single host server at the same time (VMware, 2015). The virtualization environment at the university is a Virtual Desktop Infrastructure (VDI) platform hosted on servers at the university datacenter. As discussed in the Introduction, Citrix (2017) defines a Virtual Desktop Infrastructure (VDI) as the process of running a user desktop inside a virtual machine that resides on a server in a datacenter. It is a form of virtualization that enables personalized desktops for each user to be run within a virtual machine (VM), but with all the security and simplicity of centralized management.

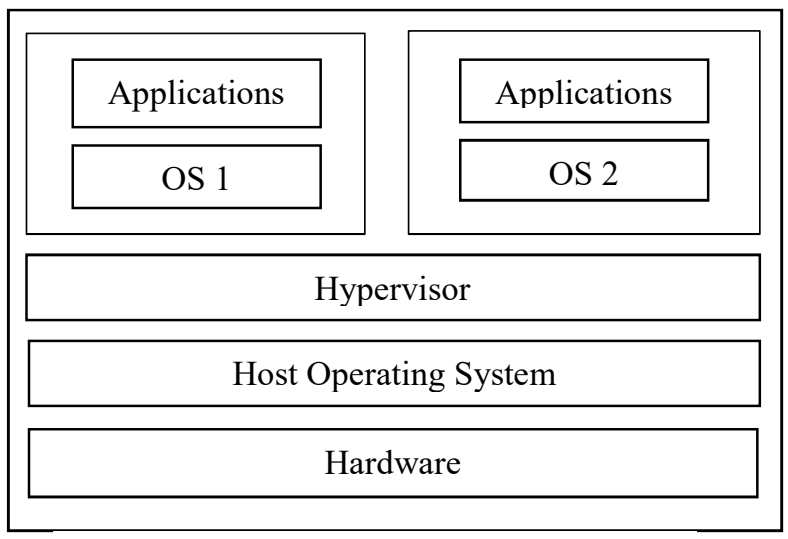

Figure 1. Type 2 Hypervisor

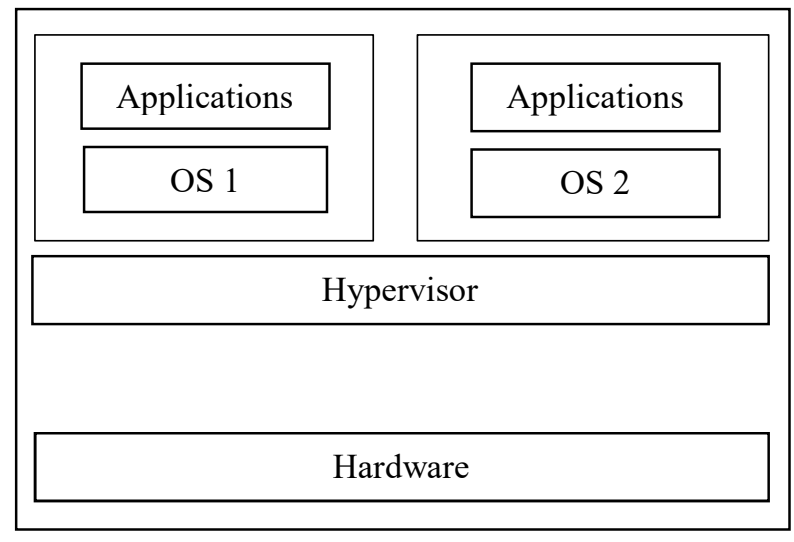

Figure 2. Type 1 Hypervisor

Bauer, E., \& Adams, R. (2012) describe two types of hypervisors: Type 1 and Type 2. Figure 1 shows a Type 2 hypervisor where the hypervisor runs on top of an existing host operating system to monitor the individual VM operating systems (i.e., the "guest" operating systems). Figure 2 shows a Type 1 hypervisor which runs directly on the hardware and monitors the guest operating systems which are a level above the hypervisor.

The VMWare Horizon with View product (VMWare, 2017 -a) used to support the courses and described in the previous section was running a Type 1 hypervisor as shown in Figure 2. The operating systems in the individual virtual machines were running Windows 7 and the applications installed on the various virtual machines varied from class to class and included Microsoft Visual Studio and Microsoft SQL Server 2008 or Netbeans and MySQL.

\section{LITERATURE REVIEW}

Virtual machines have been used in teaching environments to teach classes in networking, network security, system administration (Marshall, B., Cardon, P., Callender, C., Robertson, and P, Patel, N., 2011), operating systems, database systems and administration, distance education (Stackpole, B., 2008; Stackpole, B., Koppe, J., Haskell, T., Guay, L., and Pan. Y., 2008), system architectures (Duignan, S., \& Hall, T., 2008) and other teaching areas (Ogunyemi, A. and Johnston, K., 2010; Bower, T., 2010; and Hacker, T., 2011). Some of these teaching environments used virtual machines installed on individual computers while others used server-based virtualization technology. The following reviews summarize in more detail how virtualization technology has been used in the teaching of information technology and programming.

Grammer, Stolerman \& Yi (2015) describe the use of virtualization technology at Salem State University on students' own personal computers in an undergraduate operating systems course. The course introduced virtualization to the students who were required to install Linux on the VM. The students learned about the installation of an operating system, the command line interface, updating the Linux kernel, and system calls. A survey was conducted regarding 
the structure of the course and the use of the virtualization technology to which $80 \%$ of the students responded favorably.

Nieh and Vaill (2005) also describe the use of virtualization technology at Columbia University in an Operating Systems course for Linux kernel development. The course used the VMWare Workstation virtual machine software on which the Linux operating system was installed to provide the environment on which students could do kernel development projects. By using this environment, students did not have to worry about crashing the operating system where they made changes to the kernel code. A fresh virtual machine could be easily restarted if the operating system crashed. They found that the experiences of both students and instructors using this approach to teaching operating systems have been very positive and have been deployed to teach over 900 students effectively.

Griffin \& Jourdan (2012) present an overview of virtualization technology where both desktop and server-based VDIs have been used in university education. They describe that at the College of Engineering at Colorado State University, virtualization has been successfully used to create a variety of electronic classrooms, meeting rooms, and virtual labs. They also describe the use of virtualization technology at North Carolina State University and East Carolina University who partnered with IBM to create an online system for extending resource-intensive labs used in computer technology courses to the homes of students.

Li (2010) describes the use of VMWare and VirtualBox virtualization technology for information and computer technology courses at East Carolina University. Courses in Intrusion Detection Technologies, Scripting for Information Technology, and Network Environment II used desktop virtual machines. The Intrusion Detection course performed exercises where a server with intrusion detection software interacted with an attacking machine. The Scripting for Information Technology course had the students do programming projects where they wrote PHP scripts and performed server administrative tasks on a Linux system running in a virtual machine. In the Network Environment course students ran a script on a virtual machine to establish a VPN tunnel with a central server on campus. They found that both VirtualBox and VMware were viable virtualization solutions for educational purposes.

Ma, Teng, Du and Zhang (2005) describe the use of cloud-based virtualization technology at the University of Jinan in China for an undergraduate Software Engineering course to teach students project-driven, learning-by-doing team software development in Java. They found that their experiment of using a cloud-based virtualization platform in the course worked well.

Villanueva and Cook (2005) describe the use of the VMWare GSX Server virtualization platform to support Networking Systems Administration courses. The platform ran on two servers and was available 24/7 to all students in the courses via internet access both on-campus and off-campus. They were successful in configuring the platform to successfully isolate the VMs from the rest of campus and still provide the course with the needed requirements to teach DHCP, DNS and WINS services. Even though they found that there were a finite number of VMs that could run at any given time, the project was considered successful.

Mirzoev, T. (2014) describes the use of a virtualization technologies to teach Introduction to Information Technology (students learn DHCP, DNS, Linux storage systems), and Datacenter Management (students learn virtualization technology and cloud computing. Fifteen Dell R610 servers running VMWare ESX 4.1 operating systems are used to support the Datacenter Management course and fifteen IBM x335 servers are used for the Introduction to Information Technology freshmen course which support at least 60 students. The use of this technology allowed students to learn virtualization technology and received the opportunity to learn many aspects of IT systems to the full extent possible. They recommend based on their experience that appropriate commercial training on virtualization and network storage be implemented for faculty teaching the courses, and that a teaching assistant, specifically a student who has taken the Datacenter Management course be certified in virtualization technologies.

Lunsford, D. L. (2009) describes the use of virtualization in an information systems security course to enable coverage of high-risk computer security topics with the focus on the examination of security measures, creation and implementation of policies for information assurance, and desktop security. VMWare Workstation 6 was selected as the virtualization platform since it provided both server and desktop virtualization. Students created a virtual machine on the lab computer and installed Microsoft Windows XP Service Pack 1 on it. Students served as the administrators of their own virtual machines which allowed them to do things on the virtual machines that they could not do on the host computers such as installing updates to Windows XP, creating user accounts, user directories, installing various 
security applications, and customizing system settings. Students expressed satisfaction with the course and the use of virtual machines and commented that the use of hands-on activities helped them to better understand the material presented in lectures. Since the students did custom configuration to their environments there were some problems for the IS instructor in monitoring the students' activities and assisting students in solving some problems.

Wang, X., Hembroff, G. C., \& Yedica, R. (2010, October) describe a 24/7 remotely accessible virtual laboratory environment using VMware vCenter Lab Manager and VMware vSphere vCenter on a load-balanced cluster of eight ESX 3.5 servers to conduct hands-on laboratory experiments in undergraduate education for computer security and system administration. Guest operating systems ran on VMware ESX host. The virtual machines were created by students from a pool of VM templates which defined the configuration of a virtual machine, including its hardware specification, guest operating system and network connection.

Garcia, C. R., Quesada, A., Candela, S., Carrasco, E., \& Gonzalez, A. (2012, April) describe the use of virtualization and hosting technologies to support Fundamentals of Operating Systems and Information System Technologies courses. The technologies used were Red hat Cluster Suit for clustering, Xen for virtualization, VMLB software for virtual machines load balancing and the data center manager OpenQRM for cloud computing. The Operating System Administration Lab had to support 150 students and had a cluster with 12 active nodes to provide the virtualization service, each cluster node had a processor with 4 cores, 8 Gbytes of RAM and 1 Gigabits network interface. Students performed system administration practices activities on their individual virtual machines which ran Linux. The Information Systems Technologies Virtual Lab used the same cluster in support of 25 students were the students performed activities of high availability, security, virtualization, and cloud computing.

The background review found that the use of virtualization technology in support of information technology education has been extensively used, but was not used substantially to support programming courses. This study describes how virtualization was used to support programming courses and the experiences of using such an environment specifically as a programming environment.

\section{STUDY QUESTIONS, PARAMETERS, AND LIMITATIONS}

\section{Study Questions}

1) What type of issues / complaints are encountered when using a server-based VDI platform in programming classes?

2) What is the frequency of incidents for each of the issue / complaint types that are encountered when using a server-based VDI platform in programming classes?

3) How many students are impacted by issues / complaints when using a server-based VDI platform in programming classes?

4) What percentage of students are impacted by issues / complaints when using a server-based VDI platform in programming courses compared to the percentage of students impacted by issues / complaints when configuring their own computers with the required software for specific courses?

5) How do the various issue / complaints break down proportionately as compared to all the issues?

\section{Parameters}

The parameters that are measured to answer the study questions are: 1) I: the frequency of VDI issues / complaints by category for each class, 2) S: the number of students in each class, and 3) C: the number of students that submitted VDI issues / complaints per category of issue.

\section{Limitations and Course Descriptions}

The study is limited to two courses: 1) CNIT 350 Object Oriented Programming: a 3-credit undergraduate, advanced object-oriented programming course using Java and the MySQL database system, and 2) CNIT 255 Internet Programming: a 3-credit undergraduate dynamic web programming course using ASP.NET and the SQL Server 
database system. These two courses were selected because both were programming courses and were the same courses used in a previous study by Stefanek \& Patel (2015) where similar data was collected from students that configured their own computers with the required software. By using the same courses at the same institution with the same professor, the data between the two studies could be compared to determine if there were any advantages to using one programming environment over another.

The object-oriented programming course taught Java programming which included writing code that selected, updated, inserted, and deleted data from a MySQL database system. A team project in the course involved writing a real-world like application for a customer relationship management where students used Java Swing to implement a graphical user interface to selected, display, update and insert records from and to the MySQL database system.

The internet programming course taught ASP.NET programming which included writing code that selected, updated, inserted, and deleted data from a SQL Server database management system. A team project in the course involved writing a web store application using ASP.NET forms to select, display, update and insert a variety of records from and to the SQL Server database management system.

The total number of participants in the study were 100 students. The number of student participants in the study is broken out by course and are shown in Table 1 .

Table 1. Participants in the Study

\begin{tabular}{|l|l|l|}
\hline Course & Semester / Year & Number of Students (S) \\
\hline CNIT 350: Object Oriented Programming & Spring 2015 & 14 \\
\hline CNIT 255: Internet Programming & Fall 2015 & $20^{*}$ \\
\hline CNIT 350: Object Oriented Programming & Spring 2016 & 30 \\
\hline CNIT 255: Internet Programming & Spring 2016 & 7 \\
\hline CNIT 255: Internet Programming & Fall 2016 & 12 \\
\hline CNIT 350: Object Oriented Programming & Spring 2017 & $17^{*}$ \\
\hline TOTAL & & $\mathbf{1 0 0}$ \\
\hline
\end{tabular}

* VDI used after mid-semester.

\section{VIRTUALIZATION ENVIRONMENT DESCRIPTION IN SUPPORT OF PROGRAMMING COURSES}

An approach that was initially considered to get around the problems described above was to create VMs which had all the required software already installed. These preconfigured VMs could be distributed to each student in the class which they could run on their own personal computers. The drawback to this approach was that students needed to install virtual machine software on their computer (e.g., Oracle Virtualbox or VMWare Workstation) to run the VM. This approach required the student to own a computer and to have the adequate hardware requirements to support a VM (i.e., enough RAM, virtualization turned on in BIOS, enough disk space).

To get around this problem, a server-based Virtual Desktop Infrastructure (VDI) platform was used that was hosted in the university IS datacenter and made available to students for specific courses requesting access. By using a serverbased VDI platform, users did not have to setup and run their own VMs on their personal computers. Specifically, a VDI platform refers to the infrastructure for running a user desktop inside a virtual machine that resides on a server in a datacenter with all the security and simplicity of centralized management (Citrix, 2017). By having this type of environment, each student had access to their own VM on the platform which was preconfigured with the required software for the specific programming course and available 24/7. Students needed an internet connection to access the VDI platform and could access it from home using their personal computer or by using the university lab computers. Therefore, the environment necessary for each programming course was setup and available to all students in the class without the student having to configure their own environment and was accessible to students that did not have their own personal computers.

Specifically, the VMWare Horizon with View (VMWare, 2017 -a) product was used as the virtualization platform (Type 1 hypervisor). The operating system for the virtual desktops was Windows 7 and the applications installed on the VDI included Microsoft Visual Studio and Microsoft SQL Server 2008 for the Internet Programming course (CNIT 
255) and Netbeans and MySQL for the Object-Oriented Programming course (CNIT 350). Each VDI was allocated 2-4GB of RAM and there were 12-20 virtual desktops created in the Fall semesters and 14-38 virtual desktops created in the spring semesters. The data center had a cluster of 5 servers with a dual Xeon processor and a total of 165 GB of total memory.

\section{VDI Desktop Configuration}

Master virtual machines were configured with the required software for each course by either the instructor or lab technician. The master virtual machines were hosted on servers in the IS datacenter running the VMware Horizon product. Each course had its own master virtual machine configured with the required software:

- CNIT 350: The object-oriented programming course used Java SE, the Netbeans IDE, the MySQL database management system, and MySQL Workbench.

- CNIT 255: The internet programming course used Microsoft Visual Studio 2012 (ASP.NET programming using VB or C\#), the Microsoft SQL Server 2008 database management system, and SQL Server Management Studio.

Once the master VMs were configured for each course, then these VMs were provided to the university IS datacenter staff that were responsible for the virtualization environment. The IS department created individual VMs from the master VMs for each student in each course. Each of the individual VMs were hosted on the IS department's VDI platform server(s) running the VMWare Horizon product. Each individual virtual machine was allocated from $2-4$ GB. The VMWare Horizon product makes a virtual desktop available through a single Virtual Desktop Infrastructure (VDI) virtualization platform in or to make management easier for the IS staff and accessibility easier to users (VMWare -a, 2017).

Stefanek \& Patel (2015) discuss the various steps that the IS department needed to go through to plan for hardware and software needed to support a VDI virtualization platform for the requesting academic departments' needs. The academic department typically notified the IS datacenter staff at the end of the semester whether they would have a need for the VDI platform during the next semester and provided an estimate of the number of students that would need to use it. During the first week of the next semester, each instructor provided a list of students in the class (email addresses) to the VDI infrastructure administrator. The VDI infrastructure administrator setup the environment for student access by the start of class.

Students were required to have an internet connection to access the VDI platform. They could access the VDI by downloading a VMWare Horizon client software component onto their desktop and then use the client to connect to the VDI. The user enters a connection server in the Horizon client popup window to connect to the VDI platform (e.g., university-horizon.pnw.edu). The user initiates the connection to the connection server and is prompted to enter their authentication information. After the user enters their individual authentication information, one or more icons appear that describe the VDI for the course (e.g., CNIT350 and/or CNIT255). The user clicks on the course icon and the VDI is booted up. Once the VDI is booted, the student can begin using it like any local computer. Typically, the VDI will have USB port access and access to university virtual drives so that students can copy their programs from the VDI onto a flash drive or their virtual drive.

\section{METHODS}

The methods used for data collection were: 1) reporting issues / complaints by email, and 2) reporting issues / complaints in-person. Students were instructed at the start of each course to report any issues they were having with the VDI by sending an email describing the issue directly to the instructor. Alternatively, they were instructed that they could report an issue directly to the instructor during class. If an issue was reported verbally, the instructor would record the issue, the date it was reported, and the student that reported it. The data collected is reported in Tables 2 and 3 . 


\section{RESULTS}

Table 2 describes the breakout of reported issues / complaints by all students. There were 13 issue / complaint categories reported. The same student could report recurring issues at separate times as separate incidents, but the same incident reported by a single student multiple times was counted as one incident.

Table 2. Frequency of Issues/Complaints when using a VDI

\begin{tabular}{|c|c|c|c|c|c|c|c|}
\hline $\begin{array}{l}\text { Issue / Complaint } \\
\text { Type }\end{array}$ & $\begin{array}{l}\text { Freq. } \\
\text { (I) } \\
\text { (CNIT } \\
\text { 350 } \\
\text { Spring } \\
\text { 2015) }\end{array}$ & $\begin{array}{l}\begin{array}{l}\text { Freq. } \\
\text { (I) }\end{array} \\
\text { (CNIT } \\
255 \\
\text { Fall } \\
2015)\end{array}$ & $\begin{array}{l}\text { Freq. } \\
\text { (I) } \\
\text { (CNIT } \\
\text { 350 } \\
\text { Spring } \\
\text { 2016) }\end{array}$ & $\begin{array}{l}\text { Freq. } \\
\text { (I) } \\
\text { (CNIT } \\
255 \\
\text { Spring } \\
2016)\end{array}$ & $\begin{array}{l}\text { Freq. } \\
\text { (I) } \\
\text { (CNIT } \\
255 \\
\text { Fall } \\
2016)\end{array}$ & $\begin{array}{l}\text { Freq. } \\
\text { (I) } \\
\text { CNIT } \\
350 \\
\text { Spring } \\
\text { 2017) }\end{array}$ & Total \\
\hline VDI Time set Incorrectly & 1 & & & & & & 1 \\
\hline VDI Not Running Genuine Windows & 1 & & & & & & 1 \\
\hline VDI Slow / During Login & 1 & & 1 & & & & 2 \\
\hline VDI Slow / During Use & 1 & & 8 & & & 4 & 13 \\
\hline $\begin{array}{l}\text { VDI Can't Access / } \\
\text { During Login / VDI Busy }\end{array}$ & & & 3 & & & & 3 \\
\hline $\begin{array}{l}\text { VDI Can’t Access / } \\
\text { During Login / Timeout }\end{array}$ & & & 4 & & & & 4 \\
\hline VDI Can't Access / Can't Resolve Host & & & & & 1 & & 1 \\
\hline VDI Can’t Access / Not Available & 5 & & & & 1 & 2 & 8 \\
\hline $\begin{array}{l}\text { VDI Can't Access / University Virtual } \\
\text { Drives }\end{array}$ & & & 1 & 2 & & & 3 \\
\hline VDI Can’t Access / USB Drive & & & & 1 & & & 1 \\
\hline Incompatible Databases between VDIs & & & & & 1 & & 1 \\
\hline Database not Started during Boot & & & & & 1 & 3 & 4 \\
\hline Don't Know How to Access VDI & 1 & & 1 & & 2 & & 4 \\
\hline Total & 10 & $\mathbf{0}$ & 18 & 3 & 6 & 9 & 46 \\
\hline
\end{tabular}

Table 3 breaks out the number of individual students that were affected by the issues in Table 2. Even though a student may have had multiple issues, the student will be recorded as a single student affected by the issues.

Table 3. Students Impacted by All Issues / Complaints by Class

\begin{tabular}{|l|l|l|}
\hline Type of Course & Students Affected (C) / Total Students & Percentage \\
\hline CNIT 350: Object Oriented Programming & $28 / 61$ & $45.9 \%$ \\
\hline CNIT 255: Internet Programming & $3 / 39$ & $7.7 \%$ \\
\hline $\begin{array}{l}\text { Total Students Affected Across All Courses / All } \\
\text { Issues }\end{array}$ & $31 / 100$ & $31 \%$ \\
\hline
\end{tabular}

\section{ANALYSIS}

The number of different issues / complaints encountered when using a VDI for programming courses was 13 as described in column 1 (Issue / Complaint Type) in Table 2. The most frequent set of issues / complaints were those related to students not being able to access the VDI. The assorted reasons reported for not being able to access the VDI were: 1) the connection timed out, 2) the VDI was busy, 3) the logon attempt could not resolve host, and 4) the VDI was not available. The total number of issues / complaints regarding "VDI Can't Access", related to not being able to access the VDI, are 16 / 46 or $34.8 \%$ of the issues. The exact technical explanations why the VDI could not be 
accessed are not known. The VDI timing out on login and being busy may be the same issue, but reported with different terminology by the students. The timeout issue and the VDI platform "Not Available" may have been due to network or other technical reasons that prevented access to the VDI platform.

The second most frequently reported issues / complaints (Table 2) were that the virtualization environment ran slow (i.e., "VDI Slow") during use (13 / 46) and during login (2 / 46). The total number of issues / complaints regarding the virtualization environment being slow was $15 / 46$ issues or $32.6 \%$ of all issues. This issue category was reported widely by a variety of students. It was also reported by eight students that the VDI was slower off-campus than oncampus. A possible explanation for this difference may be due to a faster internet connection on campus than the typical internet connection at home. Nevertheless, the VDI was considered slow as reported by $32.6 \%$ of the students either on or off campus. Some of the potential contributing factors to the slow environment may have been: 1) most students had a VDI with a 2 GB limit of RAM which may have been too little RAM, 2) there may have been too few servers to host all the students within the virtualization environment, and 3) the virtualization software may have needed to be fine-tuned / configured to meet the needs of the class load.

The next set of categories of issues / complaints all had the same number of incidents reported: 1) not being able to access external drives, 2) the database management system service was not starting during boot of the VDI, and 3) students not knowing what to do. The number of incidents in each category were 4 / 46 or $8.7 \%$.

Table 3 shows that $31 \%$ of all students using the virtualization environment for their courses experienced at least one of the issues described in Table 2. This compares to $29.2 \%$ of all students experiencing issues when configuring their own computers with IDEs and DBMSs as described by Stefanek \& Patel (2015) in a previous study in the same classes at the same institution using the same IDEs and DBMSs as in the current study. In the previous study, there were 24 out of 82 students (29.2\%) affected by either installation, configuration, incompatibility or integration issues in the same two classes.

The breakout in Table 3 shows that there was a much larger percentage of students in CNIT 350 - Object Oriented Programming (45.9\%) affected by virtual machine issues using the virtualization environment, than students in CNIT 255 - Internet Programming (7.7\%). Both virtual machine environments used an IDE and a database management system. It cannot be concluded why there were differences between the two course VDIs. There were no notable differences in the virtualization hardware. The software between the two VDIs differed in that CNIT 255 used Microsoft Visual Studio and the Microsoft SQL Server database management system while CNIT 350 used Java, the Netbeans IDE and the MySQL database management system which are all comparable products.

\section{CONCLUSIONS}

It can be concluded that there was no significant difference in the number of students experiencing issues, $31 \%$ (statistic from this study) vs. 29.2\% (statistic derived from previous study by Stefanek \& Patel (2015)) using a virtualization environment as opposed to students using their own computers configured by themselves with all the required software in the same two courses (CNIT 350: Object Oriented Programming and CNIT 255: Internet Programming). A similar quantity of issues were experienced between the two studies, but the issues were of a different type.

The advantages of using a server-based VDI platform were: 1) the configuration problems experienced when students configured their own computers with the required software were eliminated, 2) the incompatibility issues when integrating software components developed on separate computers were eliminated, 3) students had 24/7 access to the VDI both on-campus and off-campus, and 4) students could use computers that did not have the hardware requirements for running a VM locally.

The disadvantages of using a server-based VDI platform were: 1) students occasionally had trouble accessing the VDI and 2) the VDI performance was generally described as slow.

In conclusion, despite the issues encountered using the server-based VDI platform the author would recommend the use of this environment because it may be possible to improve the performance and availability of the virtualization 


\section{Issues in Information Systems \\ Volume 18, Issue 3, pp. 184-194, 2017}

environment through potential hardware improvements, increased RAM per VDI, possible virtualization configuration tweaks, and timely support of the environment which could eliminate or reduce up to $67.4 \%$ (34.8\% ("VDI Can't Access") $+32.6 \%$ ("VDI Slow")) of the issues related to using the virtualization infrastructure at the university.

Also, the use of the VDI platform allowed students to have access to the programming environment remotely and onsite even if they did not own a computer or have a computer with under configured hardware. Given, that some, although very few students do not own a computer or have a computer with adequate hardware, it is necessary to make a programming environment available to students in more complex programming courses that use a database management system. This approach is a valid approach that has worked reasonably well in the two courses described in this paper. The strategy of giving each student a pre-configured virtual machine (VM) which a student can copy onto their own computer is a valid alternative, with the drawback that large VMs may take a long time to copy back and forth between the computer and a flash drive.

\section{REFERENCES}

Bauer, E., \& Adams, R. (2012). Reliability and availability of cloud computing. John Wiley \& Sons.

Bower, T. (2010). Experiences with Virtualization Technology in Education. Journal of Computing Sciences in Colleges, 25(5), 311-318.

Citrix. (2017). What is VDI. Retrieved from https://www.citrix.com/glossary/vdi.html.

Distributed Management Task Force. (2010, January 12). Open Virtualization Format Specification, Version 1.1.0. DSP0243.

Duignan, S., \& Hall, T. (2008). Using Platform Virtualisation to Teach System Architectures in Undergraduate Computer Science-An Evaluation of Student Learning Experiences. Innovative Techniques in Instruction Technology, E-learning, E-assessment, and Education, 479-484.

Garcia, C. R., Quesada, A., Candela, S., Carrasco, E., \& Gonzalez, A. (2012, April). Using a virtual and hosted lab for information systems technologies. In Global Engineering Education Conference (EDUCON), 2012 IEEE (pp. 1-9). IEEE.

Grammer, K., Stolerman, J., \& Yi, B. (2011). Introduction of virtualization in the teaching of operating systems for CS undergraduate program. Journal of Computing Sciences in Colleges, 26(6), 44-50.

Griffin III, T. F., \& Jourdan, Z. (2012, March). Educational use cases for virtual machines. In Proceedings of the 50th Annual Southeast Regional Conference (pp. 365-366). ACM.

Hacker, T. (2011). AC 2011-1231: Exploring the Use of Virtual Machines and Virtual Clusters for High Performance Computing Education. American Society for Engineering Education. Available: www.asee.org/public/conferences/1/papers/1231/download

Li, P. (2010). Selecting and using virtualization solutions: our experiences with VMware and VirtualBox. Journal of Computing Sciences in Colleges, 25(3), 11-17.

Lunsford, D. L. (2009). Virtualization technologies in information systems education. Journal of Information Systems Education, 20(3), 339.

Ma, K., Teng, H., Du, L., \& Zhang, K. (2014). Project-Driven Learning-by-Doing Method for Teaching Software Engineering using Virtualization Technology. iJET, 9(9), 26-31. 
Marshall, B., Cardon, P., Callender, C., Robertson, and P, Patel, N. (2011). Using VMware vCenter to Teach System Administration in a Lab. Issues in Information Systems, 12(2), 153-161.

Mirzoev, T. (2014). Employing Virtualization for Information Technology Education. arXiv preprint arXiv:1404.2167.

Nieh, J., \& Vaill, C. (2005, February). Experiences teaching operating systems using virtual platforms and linux. In ACM SIGCSE Bulletin, 37(1), 520-524.

Ogunyemi, A. and Johnston, K. (2010). The use of virtual machines to support hands-on learning experiences in undergraduate systems-oriented courses. Proceedings of the 4th International Conference on Dynamic Informatics, Monash University, South Africa.

Oracle. (2017). Oracle VM Virtualbox. Available: https://www.oracle.com/virtualization/virtualbox/index.html

Shields, G. (2008). The Shortcut Guide to Selecting the Right Virtualization Solution. Realtime Publishers.

Stackpole, B. (2008). The Evolution of a Virtualized Laboratory Environment. Proceedings of SIGITE 2008, 243248.

Stackpole, B., Koppe, J., Haskell, T., Guay, L., and Pan. Y. (2008). Decentralized Virtualization in Systems Administration Education. Proceedings of SIGITE 2008, 249-253.

Stefanek, G., \& Patel, N. (2015). MOVING TOWARD A SERVER-BASED VIRTUAL MACHINE HOSTING ENVIRONMENT IN SUPPORT OF UNIVERSITY INFORMATION TECHNOLOGY PROGRAMMING COURSES. Issues in Information Systems, 16(1).

Villanueva, B., \& Cook, B. (2005, November). Providing students 24/7 virtual access and hands-on training using vmware GSX server. In Proceedings of the 33rd annual ACM SIGUCCS conference on User services (pp. 421-425). ACM.

VMware. (2015). What is a Virtual Machine: VMWare. Available: http://www.vmware.com/virtualizationbasics/how-virtualization-works.html

VMWare. (2017 -a). Horizon 7. Available: http://www.vmware.com/products/horizon.html

VMWare. (2017 -b). Virtualization. Available: http://www.vmware.com/solutions/virtualization.html.

VMWare. (2017 -c). Workstation for Windows. Available: https://www.vmware.com/products/workstation.html

Wang, X., Hembroff, G. C., \& Yedica, R. (2010, October). Using VMware VCenter lab manager in undergraduate education for system administration and network security. In Proceedings of the 2010 ACM conference on Information technology education (pp. 43-52). ACM. 